- Original Article

\title{
Potentially Inappropriate Medications by Beers Criteria in Older Outpatients: Prevalence and Risk Factors
}

\author{
Yeon-Jung Lim, Ha-Yeon Kim, Jaekyung Choi*, Ji Sun Lee, Ah-Leum Ahn, Eun-Jung Oh, Dong-Yung Cho, \\ Hyuk-Jung Kweon \\ Department of Family Medicine, Research Institute of Medical Science, Konkuk University Medical Center, Konkuk University School of Medicine, \\ Seoul, Korea
}

Background: Prescription of inappropriate medicine to elderly patients is a major public health care concern. The Beers criteria have been commonly employed as a screening tool to identify the use of potentially inappropriate medications (PIMs). The present study investigated the prevalence of PIM use according to the Beers criteria as well as factors related to PIM use.

Methods: Data obtained from a retrospective survey included 25,810 outpatients aged $\geq 65$ years from a university medical center in Seoul, Korea. PIMs were defined using the Beers criteria. Factors associated with PIM use were evaluated using multiple regression analysis.

Results: Of all participants, 7,132 (27.6\%) were prescribed at least one PIM. The most commonly prescribed PIMs were alprazolam (11.2\%), clonazepam (10.8\%), zolpidem (8.7\%), quetiapine (8.4\%), and hydroxyzine (5.4\%). In multivariate logistic regression analysis, having five or more prescription medicines (odds ratio [OR], 11.32; 95\% confidence interval [CI], 9.38 to 13.66) and five or more prescribing doctors (OR, 4.40; 95\% CI, 3.59 to 5.39) were strongly associated with PIM. In a likelihood ratio test for trend, an increasing number of medications and prescribing doctors were both significantly associated with PIM.

Conclusion: At a university medical center, the number of medications and the number of prescribing doctors was associated with PIM in older outpatients.

Keywords: Beers Criteria; Potentially Inappropriate Medication List; Aged; Prescriptions 


\section{INTRODUCTION}

There has been an unprecedented worldwide increase in the number of elderly individuals, and this has been referred to as 'a rapidly aging world. ${ }^{1)}$ It is projected that by the year 2030 , a quarter or more of the US population will contain people aged $\geq 65$ years, and globally, the elderly population will reach $\geq 1$ billion by the year $2050 .^{2)}$ The population of South Korea is also rapidly aging, and as reported by Statistics Korea, the population aged $\geq 65$ years comprised $13.1 \%$ of the Korean population in 2015 . This proportion is expected to increase to $24.3 \%$ by the year 2030, and $37.4 \%$ by the year $2050{ }^{3)}$

Many elderly people have various chronic diseases and require a number of medicines. It is estimated that some of these medications are potentially inappropriate and may initiate adverse events, or may cause detrimental drug interactions. In South Korea, the prevalence of chronic diseases in elderly patients was $90.9 \%$, and $73 \%$ of the elderly patients had more than two chronic diseases. ${ }^{4)}$ In addition to the harm inflicted, adverse drug events can create substantial costs for health care organizations and systems. ${ }^{5)}$ Therefore, medical treatment of the elderly patients needs to be carefully monitored and supervised.

To identify potentially high-risk medicine use by older people, in 1991, Beers et al. ${ }^{6)}$ developed the first explicit criteria that focused exclusively on nursing home residents. These criteria have been subsequently updated and/or expanded several times and are widely used by different health care systems for adults aged $\geq 65$ years irrespective of where they reside and receive medical care..$^{7-9)}$

Several previous studies have evaluated the prevalence of potentially inappropriate medications (PIMs) and their association with sociodemographic and/or health status variables included in the Beers criteria. ${ }^{10-20)}$ However, in South Korea, except for one previous study on the prevalence of Beers criteria-based PIMs in a large medical center, ${ }^{13)}$ there have been no reports on the risk factors of PIMs based on largescale medical center data. Therefore, this study aimed to determine the factors associated with PIM use as well as the prevalence of PIMs in the older outpatients at a tertiary care teaching hospital.

\section{METHODS}

\section{Study Design and Setting}

This retrospective study was conducted from January 2014 to December 2014 in an 860-bed tertiary care teaching hospital at Konkuk University Medical Center in Seoul, Korea. The study population comprised of all outpatients aged $\geq 65$ years. The exclusion criteria included the use of intravenous injections and vaccines, dialysates, and diagnostic reagents as well as incomplete medication information.

\section{Data Collection}

Data were obtained from the prescription records in the electronic medical record (EMR) database, and included patient age, sex, the number of prescribing doctors, number of medications, and any PIMs. The number of prescribing doctors was defined as the total number of doctors prescribing medicine to an individual patient, and the number of medications was defined as the number of medications for each patient.

\section{Study Instrument and Data Processing}

PIMs were defined as all 53 medication classes listed in the 2012 Beers criteria. Any identified PIMs were examined for each individual patient, and PIM use was identified when a patient's record contained one or more PIMs. Study subjects were subsequently categorized as those with PIM (the PIM group) and those without PIM (the non-PIM group) use.

\section{Statistical Analysis}

The association between frequency and percentage of PIMs and the patient's age, sex, the number of prescribing doctors $(1,2,3,4$, or $\geq 5$ prescribing doctors), and the number of medications ( $1,2,3$, 4, or $\geq 5$ medications) was investigated. Multivariate logistic regression analysis was used to estimate and adjust the influence of each variable on the PIM use. Statistical analyses were performed using the predictive analytics software IBM SPSS ver. 21.0 (IBM Co., Armonk, NY, USA). All tests of statistical significance were two-tailed: and significance was defined as $\mathrm{P}<0.05$ with $95 \%$ confidence intervals (CI).

\section{Ethical Consideration}

This study was conducted according to the recommendations of the Declaration of Helsinki and was approved by the institutional review board of Konkuk University Medical Center (KUH1230022).

Table 1. General characteristics of the study subjects

\begin{tabular}{lcc}
\hline & $\begin{array}{c}\text { Non potentially inappropriate } \\
\text { medication }(\mathrm{N}=18,678)\end{array}$ & $\begin{array}{c}\text { Potentially inappropriate } \\
\text { medication }(\mathrm{N}=7,132)\end{array}$ \\
\hline Sex (female) & $10,304(55.2)$ & $4,192(58.8)$ \\
Age (y) & $72.4 \pm 6.1$ & $73.9 \pm 6.5$ \\
No. of medications* & & $122(1.7)$ \\
1 & $2,584(13.9)$ & $313(4.4)$ \\
2 & $2,690(14.4)$ & $440(6.2)$ \\
3 & $2,576(13.8)$ & $474(6.6)$ \\
4 & $2,137(11.4)$ & $5,783(81.1)$ \\
$\geq 5$ & $8,691(46.5)$ & \\
No. of prescribing doctors ${ }^{\dagger}$ & & $3,597(50.4)$ \\
1 & $13,700(73.3)$ & $1,870(26.2)$ \\
2 & $3,491(18.7)$ & $887(12.4)$ \\
3 & $1,022(5.5)$ & $443(6.2)$ \\
4 & $324(1.7)$ & $335(4.8)$ \\
$\geq 5$ & $141(0.8)$ &
\end{tabular}

Values are presented as number (\%) or mean \pm standard deviation. Values are assessed by chi-square test for categorical variables and Student t-test for continuous variable. All P-values are $<0.001$.

*The number of medications for each patient. ${ }^{\dagger}$ The number of doctors prescribing medicine to an individual patient. 
Table 2. The twelve most commonly prescribed potentially inappropriate medications

\begin{tabular}{llrc}
\multicolumn{1}{c}{ Medications } & \multicolumn{1}{c}{ Category } & Value & Strength of recommendation \\
\hline Alprazolam & Benzodiazepine & $1,205(11.2)$ & Strong \\
Clonazepam & Benzodiazepine & $1,160(10.8)$ & Strong \\
Zolpidem & Non-benzodiazepine hypnotic & $937(8.7)$ & Strong \\
Quetiapine & Antipsychotic & $903(8.4)$ & Strong \\
Hydroxyzine & Antihistamine & $584(5.4)$ & Strong \\
Digoxin & Cardiovascular agent & $576(5.4)$ & Strong \\
Amitriptyline & Tricyclic antidepressant & $528(4.9)$ & Strong \\
Spironolactone & Angiotensin converting enzyme inhibitor & $445(4.1)$ & Strong \\
Metoclopramide & Antiemetic & $410(3.8)$ & Strong \\
Lorazepam & Benzodiazepine & $385(3.6)$ & Strong \\
Naproxen & Nonsteroidal anti-inflammatory drugs & $313(2.9)$ & Strong \\
Diazepam & Benzodiazepine & $308(2.9)$ & Strong \\
\hline
\end{tabular}

Values are presented as number of cases (\%).

*Strong: benefits clearly outweigh risks and burden OR risks and burden clearly outweigh benefits by the 2012 Beers criteria.

Table 3. Multivariate logistic regression analysis of factors associated with potentially inappropriate medications

\begin{tabular}{lcc}
\hline \multicolumn{1}{c}{ Variable } & Odds ratio $(95 \%$ confidence interval) & P for trend ${ }^{*}$ \\
\hline Sex (female) & $1.18(1.11-1.25)$ & \\
Age & $1.03(1.03-1.04)$ & \\
No. of medications ${ }^{\dagger}$ & & $<0.001$ \\
1 & $2.45(1.97-3.04)$ & \\
2 & $3.56(2.89-4.39)$ & \\
3 & $4.50(3.66-5.55)$ & \\
4 & $11.32(9.38-13.66)$ & \\
$\geq 5$ & & \\
No. of prescribing doctors ${ }^{\ddagger}$ & 1 & \\
1 & $1.17(1.09-1.25)$ & \\
2 & $1.64(1.48-1.81)$ & \\
3 & $2.51(2.16-2.93)$ & \\
4 & $4.40(3.59-5.39)$ & \\
$\geq 5$ & &
\end{tabular}

${ }^{*}$ By a likelihood ratio test for trend. ${ }^{\dagger}$ The number of medications for each patient. tThe number of doctors prescribing medicine to an individual patient.

\section{RESULTS}

Of the 25,880 total outpatients, 25,810 outpatients were enrolled in the study after excluding 70 based on the exclusion criteria described in the methods. The general characteristics of the study subjects are shown in Table 1. The mean age of the patients was $72.4 \pm 6.1$ years in the non-PIM group and $73.9 \pm 6.5$ years in the PIM group. In the nonPIM group, 55.2\% patients were women, while in the PIM group, $58.8 \%$ patients were women. A total of 7,132 patients (27.6\%) were prescribed at least one PIM.

The most commonly prescribed PIMs were alprazolam (11.2\%), clonazepam (10.8\%), zolpidem (8.7\%), quetiapine (8.4\%), and hydroxyzine (5.4\%) (Table 2). In a multivariate logistic regression analysis, female sex, older age, the number of medications, and the number of prescribing doctors were all associated with PIM use and being prescribed five or more medications (odds ratio [OR], 11.32; 95\% CI, 9.38 to 13.66). Moreover, having five or more prescribing doctors (OR, 4.40; 95\% CI, 3.59 to 5.39) was strongly associated with PIM use (Table 3). In a likelihood ratio test for trend, a higher number of medications and prescribing doctors was associated with PIM use and also significantly increased the risk of PIMs (P for trend $<0.001$ ) (Table 3).

\section{DISCUSSION}

Using the Beers criteria, the prevalence of at least one PIM in the current study was $27.6 \%$. In addition, female sex, older age, total number of medications, and number of prescribing doctors were associated with PIM use. In a previous population-level study from New Zealand, the rate of at least one PIM being prescribed based on the Beers criteria was $40.9 \%{ }^{10)}$ A study from a tertiary care teaching hospital in India reported the PIM rate as $21.86 \%,{ }^{11)}$ while a study on the hospitalized elderly patients in the US reported a PIM rate of $32.0 \%{ }^{17)}$ In a previous South Korean study, among the 652,192 outpatients at a single tertiary medical center, $5.19 \%$ received at least one PIM. ${ }^{13)}$ In addition, another study reported that $16.4 \%$ of the 163 elderly patients admitted to a general hospital were prescribed PIMs. ${ }^{15)}$ Furthermore, among the 6,347 elderly patients at a community pharmacy near a top tier general hospital, $21.4 \%$ were prescribed PIMs. However, unlike previous studies based on the Beers criteria, we identified PIMs based on the Beers criteria, the Canadian criteria, and Zhan's classification. ${ }^{14)}$ Thus, the difference between previous reports and the present study may be due to the utilization of different criteria as well as differences in the participant characteristics, available medications, and prescribing patterns. ${ }^{11)}$

Many studies have reported an increased risk of PIM with age, sex, multiple medications, and the number of co-morbidities. ${ }^{10-12,14-16,18,19)} \mathrm{In}$ Brazil, PIM use in older patients increased with increasing age and female sex. ${ }^{19)}$ In New Zealand, older age and male sex were associated with increased risk of PIM. ${ }^{10)}$ In contrast, some studies from India and Japan observed that age, sex, and number of co-morbidities were not associated with PIM use. ${ }^{11,12}$ Therefore, although these previous studies are not consistent with regard to the association of PIM with some 
factors, there is a general consensus on the association between multiple medications and PIM use. Further evidence is available from a systemic meta-analysis demonstrating that only multiple medications were positively associated with PIM use in the elderly. ${ }^{18)}$ However, in the US, older age, the presence of more chronic diseases, and a greater number of health care visits were associated with higher odds of using 1-4 medications or $>5$ medications, compared with no medications. ${ }^{16)}$ Nevertheless, only a few previous studies have evaluated the association between PIM use and the number of prescribing doctors, as we have in the current study. Seo et al. ${ }^{15)}$ observed that the risk of PIM use by older patients increased with more than two visited doctors. We, therefore, focused the present analysis on risk factors for PIM use, such as the number of prescribing doctors and being prescribed multiple medications.

An important aspect of the present study is that the analysis was conducted simultaneously for $1,2,3,4$, and $\geq 5$ medications and 1, 2, 3, 4 , and $\geq 5$ prescribing doctors. We subsequently found that an increasing number of medications and prescribing doctors were significantly associated with PIM use and that the risk of PIM use rapidly increased with $>5$ prescribing doctors and being prescribed $>5$ medications. These data underscore the necessity of a systematic drug monitoring system, and a personal physician system for the elderly patients as well as the need for each physician to be alert for PIM use in elderly patients.

In addition to the Beers criteria, the Screening Tool of Older Person's Prescriptions (STOPP) is widely used to screening medication use in the elderly. Prior to the update in 2012, the 2003 version of the Beers criteria had several limitations: it had only a simple list of inappropriate medications without classifications, and several older medications that were no longer available were included. ${ }^{13)}$ Owing to these limitations, the STOPP criteria were developed for the general elderly population in 2008 and updated in 2014..$^{13,21-23)}$ Grace et al. ${ }^{24)}$ demonstrated that the 2012 Beers criteria and the STOPP could be useful guides for physicians coordinating the long-term care of nursing home residents. Further, a study by Lee et al. ${ }^{25)}$ reported that the 2008 STOPP criteria based PIMs could detect more duplicated prescriptions and avoidable adverse drug events than the 2003 Beers criteria based PIMs. However, a Brazilian primary health care study reported that the 2012 Beers criteria identified more PIMs than the 2008 STOPP criteria. ${ }^{26)}$ Therefore, the efficacy of one criterion over another is still controversial. Furthermore, it is difficult to apply the STOPP criteria to a large-scale study due to the high level of details required. Hence, in this study, we used the 2012 Beers criteria to identify PIMs. Compared to the previous studies analyzing PIM use and the prevalence of PIM in South Korea, we included a large sample size and analyzed not only PIM use and prevalence but also associated factors. Specifically, our results showed a dose-response association between the number of prescribed medications and PIM use as well as the number of prescribing doctors and PIM use.

This study had some limitations. First, we analyzed the data based on the prescription records from the EMR system of our medical cen- ter. We were, therefore, unable to detect prescriptions from outside our medical center as well as any over-the-counter medicines. This decreases the generalizability of our findings. Second, as we used only the prescription record of the EMR, we did not have information on the general health status and co-morbidities of the patients. Thus, although PIMs have been associated with many chronic diseases, ${ }^{16,18,20)}$ we were unable to analyze associations with any disease in our study. Third, despite the widespread use of the Beers criteria for evaluating PIMs, there is considerable debate concerning the efficacy of the criteria. Moreover, to our knowledge, the Beers criteria have not been validated in South Korea, and the updated 2014 STOPP and the 2012 Beers criteria have not been compared. Fourth, as this was a retrospective study, we could not provide evidence of causality between the studied variables.

In conclusion, the number of medications and the number of prescribing doctors was associated with PIM use in outpatients aged $\geq 65$ years. To overcome the limitations of the current study, further evaluation of prevalence and risk factors for PIMs in the Korean elderly population is needed.

\section{CONFLICT OF INTEREST}

No potential conflict of interests relevant to this article was reported.

\section{ACKNOWLEDGMENTS}

This research was supported by a grant from the Korea Health Technology R\&D Project through the Korea Health Industry Development Institute, funded by the Ministry of Health \& Welfare, Republic of Korea (grant number: HI16C0526).

\section{REFERENCES}

1. DaVanzo J. Preparing for an aging world. Santa Monica (CA): RAND Corporation; 2001.

2. Goldman DP, Cutler D, Rowe JW, Michaud PC, Sullivan J, Peneva D, et al. Substantial health and economic returns from delayed aging may warrant a new focus for medical research. Health Aff (Millwood) 2013;32:1698-705.

3. Statistics Korea. Elderly statistics [Internet]. Daejeon: Statistics Korea; 2015 [cited 2016 Jan 12]. Available from: http://kostat.go.kr.

4. Chung H, Suh Y, Chon S, Lee E, Lee B, Kim K. Analysis of inappropriate medication use in hospitalized geriatric patients. J Korean Soc Health Syst Pharm 2007;24:115-23.

5. Hug BL, Keohane C, Seger DL, Yoon C, Bates DW. The costs of adverse drug events in community hospitals. Jt Comm J Qual Patient Saf 2012;38:120-6.

6. Beers MH, Ouslander JG, Rollingher I, Reuben DB, Brooks J, Beck JC. Explicit criteria for determining inappropriate medication use in nursing home residents. UCLA Division of Geriatric Medicine. Arch Intern Med 1991;151:1825-32.

7. Beers MH. Explicit criteria for determining potentially inappropriate 
medication use by the elderly: an update. Arch Intern Med 1997;157:1531-6.

8. Fick DM, Cooper JW, Wade WE, Waller JL, Maclean JR, Beers MH. Updating the Beers criteria for potentially inappropriate medication use in older adults: results of a US consensus panel of experts. Arch Intern Med 2003;163:2716-24.

9. American Geriatrics Society 2012 Beers Criteria Update Expert Panel. American Geriatrics Society updated Beers Criteria for potentially inappropriate medication use in older adults. J Am Geriatr Soc 2012;60:616-31.

10. Narayan SW, Nishtala PS. Prevalence of potentially inappropriate medicine use in older New Zealanders: a population-level study using the updated 2012 Beers criteria. J Eval Clin Pract 2015;21:633-41.

11. Pradhan S, Panda A, Mohanty M, Behera JP, Ramani YR, Pradhan PK. A study of the prevalence of potentially inappropriate medication in elderly in a tertiary care teaching hospital in the state of Odisha. Int J Med Public Health 2015;5:344-8.

12. Kondo N, Nakamura F, Yamazaki S, Yamamoto Y, Akizawa T, Akiba T, et al. Prescription of potentially inappropriate medications to elderly hemodialysis patients: prevalence and predictors. Nephrol Dial Transplant 2015;30:498-505.

13. Jang IY, Lee YS, Jeon MK, Cho H, Oh JS, Lee Y, et al. Potentially inappropriate medications in elderly outpatients by the 2012 version of Beers criteria: a single tertiary medical center experience in South Korea. J Korean Geriatr Soc 2013;17:126-33.

14. Kim A, Kim HA, Rhie SJ. Risk factors of potentially inappropriate medications and cost by polypharmacy among elderly patients of a community pharmacy near a top tier general hospital. Korean J Clin Pharm 2015;25:159-65.

15. Seo DS, Yoon JL, Kim SJ, Park JS, Lee WJ, Kim MY. Polypharmacy in older patients admitted to a general hospital. J Korean Geriatr Soc 2012;16:133-40.

16. Charlesworth CJ, Smit E, Lee DS, Alramadhan F, Odden MC. Polypharmacy among adults aged 65 years and older in the United States: 1988-2010. J Gerontol A Biol Sci Med Sci 2015;70:989-95.

17. Cartwright OM. A retrospective evaluation of potentially inappropriate medication use in hospitalized elderly patients. Wichita (KS): Wichita
State University; 2007.

18. Santos AP, da Silva DT, dos Santos Junior GA, Silvestre CC, Nunes MA, Lyra DP Jr, et al. Evaluation of the heterogeneity of studies estimating the association between risk factors and the use of potentially inappropriate drug therapy for the elderly: a systematic review with metaanalysis. Eur J Clin Pharmacol 2015;71:1037-50.

19. Baldoni Ade O, Ayres LR, Martinez EZ, Dewulf Nde L, Dos Santos V, Pereira LR. Factors associated with potentially inappropriate medications use by the elderly according to Beers criteria 2003 and 2012. Int J Clin Pharm 2014;36:316-24.

20. Seo MK, Bae MK, Lee IH, Jeon S, Yoon JH. Assessment of potentially inappropriate medication use in Korean elderly patients with Parkinson's disease. Korean J Clin Pharm 2015;25:254-63.

21. Gallagher P, Ryan C, Byrne S, Kennedy J, O'Mahony D. STOPP (Screening Tool of Older Person's Prescriptions) and START (Screening Tool to Alert doctors to Right Treatment): consensus validation. Int J Clin Pharmacol Ther 2008;46:72-83.

22. Lee WH, Won CW, Ahn JY, Choi HR, Kim BS, Kim SY. The evaluation of drug appropriacy using STOPP-START criteria for elderly inpatients at a university hospital in Seoul. Korean J Fam Pract 2014;4:157-63.

23. O'Mahony D, O'Sullivan D, Byrne S, O'Connor MN, Ryan C, Gallagher P. STOPP/START criteria for potentially inappropriate prescribing in older people: version 2. Age Ageing 2015;44:213-8.

24. Grace AR, Briggs R, Kieran RE, Corcoran RM, Romero-Ortuno R, Coughlan TL, et al. A comparison of beers and STOPP criteria in assessing potentially inappropriate medications in nursing home residents attending the emergency department. J Am Med Dir Assoc 2014;15:830-4.

25. Lee JH, Roh JH, Suh YW, Lee JH, Lee ES, Lee BK, et al. Comparison between Beers criteria and screening tool of older Person's prescriptions as a screening tool for the detection of potentially inappropriate medicines and adverse drug events in the elderly patients. J Korean Geriatr Soc 2013;17:28-36.

26. Oliveira MG, Amorim WW, de Jesus SR, Heine JM, Coqueiro HL, Passos LC. A comparison of the Beers and STOPP criteria for identifying the use of potentially inappropriate medications among elderly patients in primary care. J Eval Clin Pract 2015;21:320-5. 